\title{
Handling and Stability Analysis of Vehicle Plane Motion
}

\author{
Verbitskii Vladimir Grigorievich, Bezverhyi Anatoliy Igorevich, Tatievskyi Dmitry Nikolayevich*
}

Department of Computerized System Software, Faculty of Power Engineering, Electronics and Information Technologies, Zaporizhia State Engineering Academy, Zaporizhia, Ukraine

Email address:

oxsidan@ukr.net(V. V. Grigorievich), anatoliy.bezverkhyi@hotmail.com (B. A. Igorevich), dtdissert@gmail.com (T. D. Nikolayevich)

${ }^{*}$ Corresponding author

\section{To cite this article:}

Verbitskii Vladimir Grigorievich, Bezverhyi Anatoliy Igorevich, Tatievskyi Dmitry Nikolayevich. Handling and Stability Analysis of Vehicle Plane Motion. Mathematics and Computer Science. Vol. 3, No. 1, 2018, pp. 13-22. doi: 10.11648/j.mcs.20180301.13

Received: January 19, 2018; Accepted: February 2, 2018; Published: February 23, 2018

\begin{abstract}
The work analyzes the properties of handling and bicycle vehicle model motion stationary states manifold stability taking into account drift force nonlinear characteristics. Determining single two-axle vehicle nonlinear model stationary states and analyzing their stability were based on a graphical method (Y. M. Pevzner, H. Pacejka). It has its disadvantages: the absence of evident analytical stability criteria for the entire wheeled vehicle circular stationary states manifold. And also the absence of global stability threshold characteristics in the controlled parameter space. The task part suggests developing methods for building bifurcation manifold or critical parameters manifold (longitudinal velocity and wheel turning angle) with which the divergent loss of stability occurs. Known H. Troger, K. Zeman and Fabio Della Rossaa, GiampieroMastinub, Carlo Piccardia results are based on parameter continuation numerical methods which makes the quality analysis of drift force nonlinear characteristics impact on the entire stationary states manifold stability conditions more difficult. A compelling grapho-analytic approach towards bifurcation manifold building and getting circular stationary states analytical stability conditions based on moving from nonlinear drift forces on axles dependencies to their inverse dependence is developed in the suggested work. This methodology allows defining dangerous/safe stability threshold conditions in the control parameters space.
\end{abstract}

Keywords: Non-linear Bicycle Model, Stationary States Manifold, Vehicle Handling, Divergent Stability Loss, Parameters Bifurcation Set

\section{Introduction}

One of the vehicle dynamic properties important characteristics is the property of steering - an ability to perform a circular motion with a locus radius of curvature fixed value and continuous longitudinal velocity parameter growth [1]. Compact analytical formulas (handling equations) that determine steering properties of both linear $[1,2,3]$ and nonlinear models taking into account the non-linearity of side slip forces $[2,4,5,6,7]$ are known for a vehicle bicycle model. An important aspect when analyzing handling properties which hasn't been covered before is defining conditions for vehicle handling properties change with large enough transverse acceleration values, for example, from insufficient to excessive. Another relevant problem is determining the analytical conditions of the divergent loss of stability for the whole variety of vehicle movement circular stationary modes and its special case - the task of circular stationary modes corresponding to the handling curve divergent loss of stability. At present these tasks are solved either by using the Pevzner-Pacejkagrapho-analytical method $[6,8]$ with its advantages of simplicity and visualization, and disadvantages of missing corresponding analytic and quantitative evaluations, or building stability diagrams in the control parameters plane is done numerically based on the two-parameter continuation method which makes it more difficult to analyze the initial reasons causing the stability diagram boarders alteration (which is explained with missing corresponding analytic evaluations). Thus, the work [9] contains a complete enough analysis of a two-axle vehicle model phase portrait qualitative changes with an axles side slip forces non-linear characteristics variation and two control parameters modification complemented with numeric bifurcation diagrams building. The mentioned work doesn't 
touch upon vehicle handling properties analysis.

This article deals with handling properties of both linear vehicle model and non-linear bicycle model based on further development of the Pevsner-Pacejka grapho-analytical method complemented by a simple and effective control parameters bifurcation multitude building technique $[7,10$, $11,12]$ which to our mind can serve as a preliminary analysis test of the vehicle movement stationary states entire manifold handling and stability characteristics to evaluate various design solutions viability. More complete models can certainly be obtained involving continuation method universal numerical algorithms [13, 14].

\section{Problem State}

\subsection{A vehicle Bicycle Model with Elastic Wheels According to I. Rocard}

Let $m$ the mass and $J$ the system central moment of inertia in relation to the vertical axis, $a, b$ are vehicle centre of mass distances to the front and rear wheel axles [15].

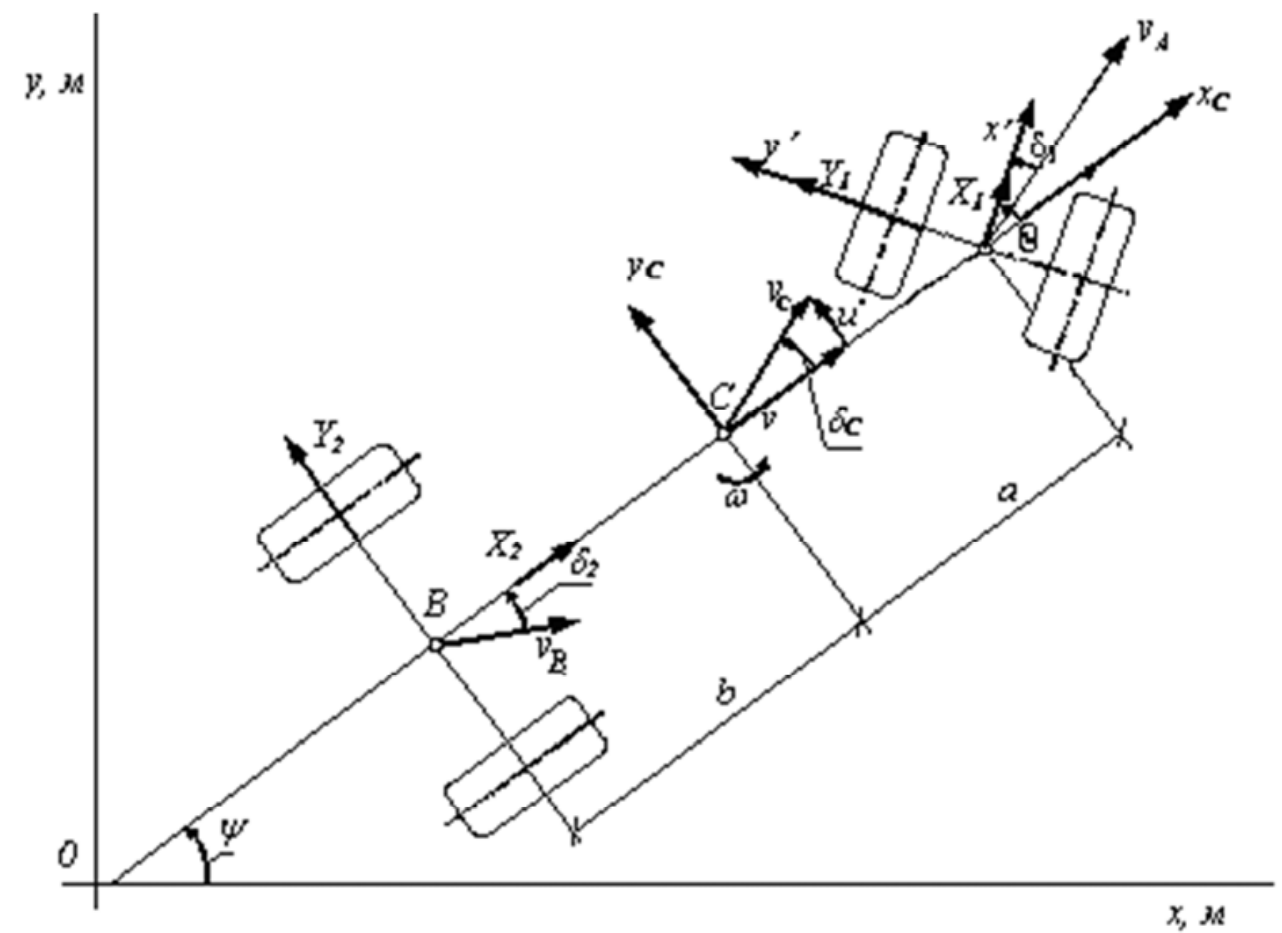

Figure 1. Vehicle with direct wheel control design model.

A vehicle bicycle model plane-parallel motion equations (1) suggesting constant longitudinal velocity $v$ look as follows $[6,16,17]$.

$$
\begin{aligned}
& m(\dot{u}+\omega v)=Y_{1} \cos \theta+Y_{2} ; \\
& J \dot{\omega}=a Y_{1} \cos \theta-Y_{2} b ; \\
& \delta_{1}=\theta-\operatorname{arctg} \frac{u+a \omega}{v} ; \delta_{2}=\operatorname{arctg} \frac{-u+b \omega}{v},
\end{aligned}
$$

where, $u-$ a vehicle centre of mass transverse component of velocity; $\omega-$ an angular velocity in relation to the vertical axis; v -a centre of mass longitudinal velocity; $\delta_{1}, \delta_{2}-$ slip angles of the front and rear axles. $Y_{i}$ slip forces are defined a posteriori and approximately represented with various analytic dependencies, for example, bearing a character of a $Y_{i}\left(\delta_{i}\right)=k_{i} \delta_{i}\left(1+\bar{k}_{i}^{2} \delta_{i}^{2} / \phi_{i}^{2}\right)^{-1 / 2}$ saturation function. The slip force is proportionate to the slip coefficient in linear approximation, $Y_{i}=k_{i} \delta_{i}, i=1,2$, suggesting that further on $\bar{k}_{i}=k_{i} / N_{i}$-side slip dimensionless ratio $\left(N_{i}\right.$-axles vertical reactions), $\bar{Y}_{i}=Y_{i} / N_{i}$-dimensionless slip forces.
A point path radius on a vehicle rolling axis the speed of which lies along the rolling axis can be represented as follows (emerges out the slip angles definition and $\mathrm{R}$ radius vector angular velocity definition):

$$
R=\frac{l}{\theta+\delta_{2}-\delta_{1}},
$$

where, $\left(\theta+\delta_{2}-\delta_{1}\right)$ is the Ackermann angle. The Ackermann angle geometric sense is the angle between radius vectors connecting the vehicle front and rear axles centres [1].

Therefore, when a vehicle is moving ( $l$ is the distance between the front and rear axles)along the $R$ radius circle with various $v$ velocity parameter values (a center of mass longitudinal velocity) the Ackermann angle should remain constant: $l / R=\theta+\delta_{2}-\delta_{1}$.

Then the front controlled wheels steering angle is defined by the following correspondence:

$$
\theta=l / R-\left(\delta_{2}-\delta_{1}\right)
$$




\subsection{The Development of Stationary Movement States Analysis Grapho-Analytical Method}

A grapho-analytical approach towards the addend defining in an expression is suggested below (3).

A set of equations defining a vehicle bicycle model stationary states manifold can be merged into a single defining equation $[6,18]$.

$$
\begin{aligned}
& -\frac{v}{g} \omega+\frac{\bar{Y}_{1}\left(\delta_{1}\right) b}{l}+\frac{\bar{Y}_{2}\left(\delta_{2}\right) a}{l}=0, \\
& \bar{Y}_{1}\left(\delta_{1}\right)-\bar{Y}_{2}\left(\delta_{2}\right)=0, \\
& \bar{Y}\left(\delta_{2}-\delta_{1}\right)=\frac{v^{2}}{g l}\left(\theta+\delta_{2}-\delta_{1}\right),
\end{aligned}
$$

Where, $\quad \bar{Y}=\bar{Y}\left(\delta_{2}-\delta_{1}\right)$ is a curve defined with dimensionless dependencies of the slip forces on axles (figure 2): $\bar{Y}=\bar{Y}_{1}\left(\delta_{1}\right)=\bar{Y}_{2}\left(\delta_{2}\right)$.

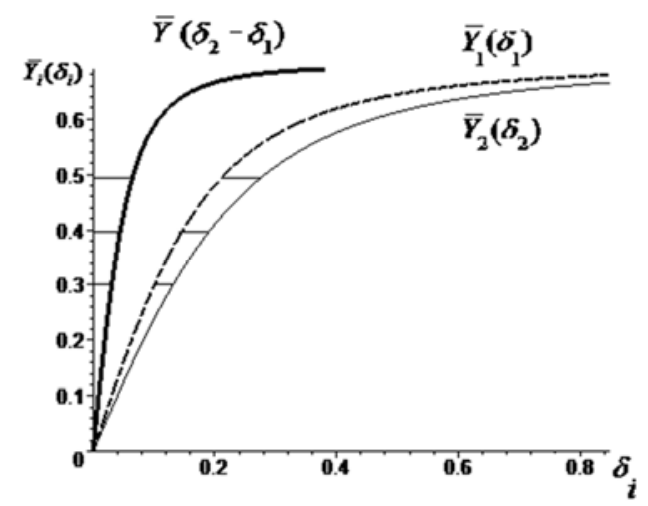

(a) plots of sideslip forces on the front and rear axles, as a function of a slip angle

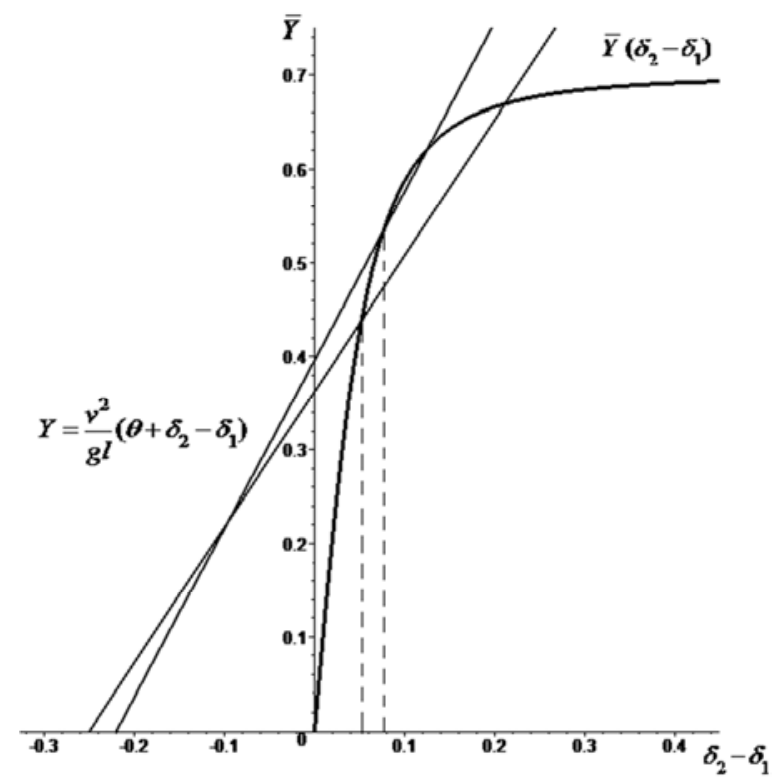

(b) stationary modes, corresponding to the movement of the characteristic point of the vehicle along a trajectory with a fixed radius of curvature $\mathrm{R}$
A movable straight line slope ratio is proportionate to the square of vehicle centre of mass longitudinal velocity $v^{2} / g l$, the controlled wheels steering angle $\theta$ defines the straight line parallel shift. Model stationary states correspond to the movable straight line and stationary curve cross points: the cross point $\bar{Y}$ ordinate defines the centre of mass specific side acceleration in the corresponding stationary state; its abscissa defines the axles slip angles difference $\left(\delta_{2}-\delta_{1}\right)$. A path with the same $R$ radius of curvature corresponds to the two stationary states with different $v$ velocity parameter value and identical Ackermann angle value (figure $4, b$ ), then the corresponding cross points ordinates are set with the $\bar{Y}=v^{2} / g R$ correlation, and the $\theta$ parameter value is received as a difference between the Ackermann angle $l / R$ and these cross points abscissa $\left(\delta_{2}-\delta_{1}\right)$ :

$$
\theta=l / R-\left(\delta_{2}-\delta_{1}\right)
$$

The corresponding $\left(\delta_{2}-\delta_{1}\right)$ difference value with this $\bar{Y}$ parameter value is defined with the $G(\bar{Y})$ function inverse to the $\bar{Y}\left(\delta_{2}-\delta_{1}\right)$ function [12].

Let's separate the $\left(\delta_{2}-\delta_{1}\right)=G(\bar{Y})$ function building stages: the $\bar{Y}_{1}=\bar{Y}_{1}\left(\delta_{l}\right), \bar{Y}_{2}=\bar{Y}_{2}\left(\delta_{2}\right)$ dependencies are the initial ones, solving them for $\delta_{i}, \delta_{l}=F_{1}(\bar{Y}), \delta_{2}=F_{2}(\bar{Y})$ are found and the required function is found as a difference between $\left(\delta_{2}-\delta_{I}\right)=F_{2}(\bar{Y})-F_{I}(\bar{Y})=G(\bar{Y})$.

After defining the $G(\bar{Y})$ function, the handling curve is received:

$$
\theta=l / R-\left(\delta_{2}-\delta_{1}\right)=l / R-G(\bar{Y}),
$$

which leads to the know handling straight line within the slip linear hypothesis:

$$
\begin{aligned}
& \left(\delta_{2}-\delta_{1}=\left(\frac{\bar{k}_{2}-\bar{k}_{1}}{\bar{k}_{1} \bar{k}_{2}}\right) \cdot \bar{Y}\right) \\
& \theta=l / R+\left(\frac{\bar{k}_{2}-\bar{k}_{1}}{\bar{k}_{1} \bar{k}_{2}}\right) a_{y} / g .
\end{aligned}
$$

All the further calculations are performed with $\bar{k}_{1}=3,3 ; \bar{k}_{2}=2,527 ; l=5 \mathrm{~m} ; v_{k p}=22,98 \mathrm{~m} / \mathrm{s} ; \phi_{1}=\phi_{2}=0,8 ;$ $R=30,5 \mathrm{~m}$ numeric parameters values, if their values are not mentioned deliberately.

Figure 3 shows the handling straight line and the handling curve demonstrating the slip force nonlinear nature influence.

It's convenient to further transit from equation (5) to equivalent equation (7) moving to inverse functions in the left and right equation (5) parts:

$$
G(\bar{Y})=g l / v^{2} \cdot \bar{Y}-\theta .
$$

Figure 2. Plots of a static curve $\bar{Y}=\bar{Y}\left(\delta_{2}-\delta_{1}\right)$ 


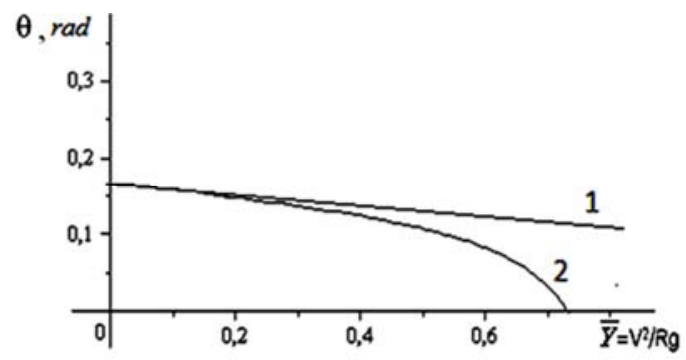

Figure 3. Steering curve.

1 - linear sideslip hypothesis $\left(\bar{Y}_{i}=\bar{k}_{i} \delta_{i}, i=1,2,\right)$;

2 - nonlinear sideslip hypothesis $\left(\bar{Y}_{i}\left(\delta_{i}\right)=\bar{k}_{i} \delta_{i}\left(1+\bar{k}_{i}^{2} \delta_{i}^{2} / \phi_{i}^{2}\right)^{-1 / 2}, \phi_{1}=\phi_{2}=0,8\right)$

Finding circular stationary states basing on the graphoanalytical approach using equation (7) suggests knowing two $\theta, v$ parameters, then the movable straight line slope should be calculated, and after defining the cross points with the stationary curve $G(\bar{Y})$, the specific side acceleration $\bar{Y}$ and $\delta_{2}-\delta_{1}$ value corresponding to the cross points is found.

In case of circular stationary states corresponding to the fixed $R$ radius of curvature, the building procedure can be simplified. Indeed, choosing the current specific side acceleration value, $\bar{Y}=\frac{v^{2}}{g R}$, one movable straight line and stationary curve cross point if definitely found, $G(\bar{Y})$ (point 1 in figure 4$)$, then point 2 is defined with $(0,-\theta)$ coordinates with a movable straight line running across it.

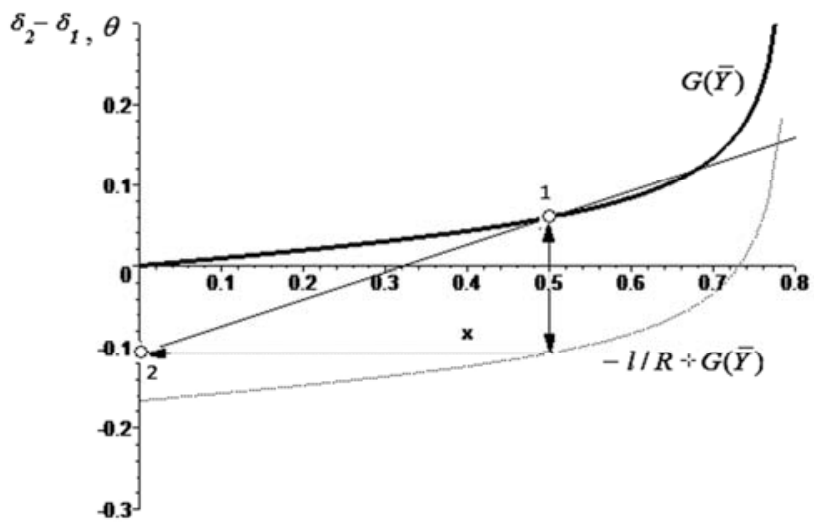

Figure 4. Illustration for the graph-analytic method of finding the stationary modes corresponding to a given value of the Ackerman angle.

It should be noted that, if $\bar{Y}=\frac{v^{2}}{g R}$ is taken as an independent argument (with the fixed $R$ value), then equation (7) is going to set handling curve (6):

$$
G(\bar{Y})=g l / v^{2} \cdot \frac{v^{2}}{g R}-\theta=l / R-\theta .
$$

\subsection{To the Analysis of a Two-Axle Vehicle Nonlinear Model Handling Properties Changing}

It derives from the handling curve diagram (figure 5) that a local minimum should appear in the first plane quarter (Y, $\left.\delta_{2}-\delta_{1}\right)$ for the $l / R-G(\bar{Y})$ function to change vehicle handling character from insufficient to excessive. The latter is related to the additional inflection points implementation; the considerable $G(\bar{Y})$ curve inflection points role is going to be discussed in the next section.

In case of slip forces approximation as monotonic dependencies bearing the character of saturation curves for a model with understeering along the linear approximation $\left(\bar{k}_{2}>\bar{k}_{1}\right)$ a required condition for moving to oversteering is $\phi_{1}>\phi_{2}$; for a model with oversteering $\left(\bar{k}_{2}<\bar{k}_{1}\right)$ a required condition to move to understeering is $\phi_{1}<\phi_{2}$ (figure 5).

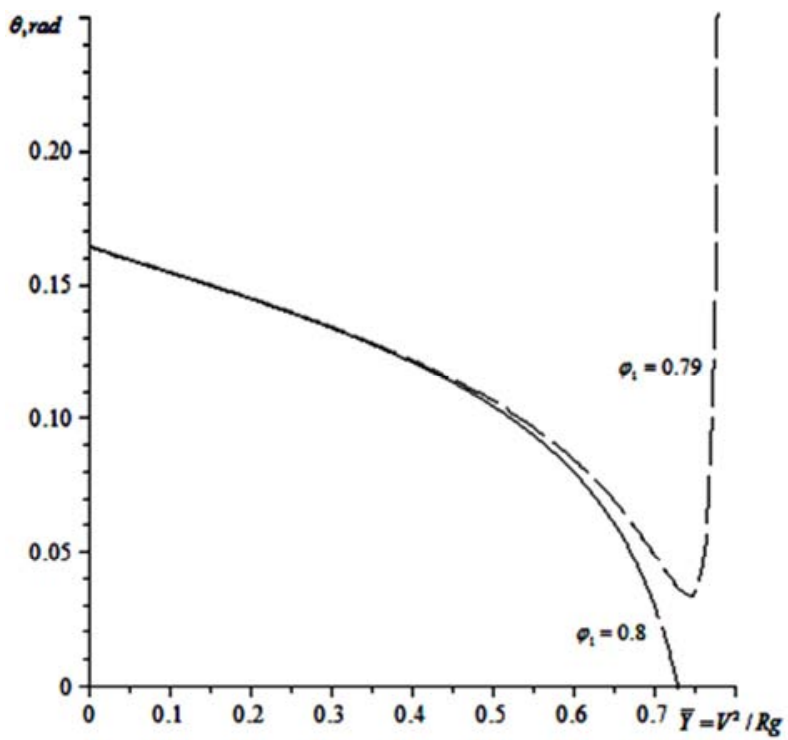

Figure 5. Steering curves when friction coefficient varies in the transverse direction on the front axle.

\subsection{The Analysis of Movement Circular Stationary States Multifold Divergent Stability Loss; Building a Bifurcation Set in a Control Parameters Plane as a Two-Value Curve to a Curve $G(Y)$}

The stationary curve $\delta_{2}-\delta_{1}=G(\bar{Y})$ and movable straight line $\delta_{2}-\delta_{1}=g l / v^{2} \cdot \bar{Y}-\theta$ cross points correspond to system (1) stationary states. Let's leave the stability defining criterion of the found stationary modes without an exhaustive grounding presenting a corresponding practitioner guide only [7, 17, 19]:

(1) A stationary state is stable if a movable plane slope is larger than a stationary curve slope in the corresponding cross point (it should be noted that this criterion is going to change the other way around when moving to a graphic representation based on equation (5) (see figure 2)).

(2) Divergent stationary state stability loss occurs only with divisible stationary state implementation - upon movable and stationary curves touching (figure 6).

The latter criterion guarantees stationary state stability properties saving with its evolving due to control parameters 
alteration up till the divisible stationary state implementation moment.

\subsection{Certain Given Criterion Argumentation}

Stationary states divergent stability loss is pertinent to zero eigen value appearing. Therefore, a linear approximation system matrix determinant numerically equal to an absolute term of a characteristic equation turns into zero. The latter fact leads to the conclusion of curves which define the stationary states manifold in the corresponding point osculation.

This way, an osculation of movable straight line and curve, $G(\bar{Y})$, corresponds to $v$ and $\theta$ parameters bifurcation values, and the entire bifurcation parameters set induces a curve reciprocal to $G(\bar{Y})$ [20]. Its parametric representation $\theta=\theta(Y), v=v(Y)$ is [12]:

$$
\begin{aligned}
& \theta=Y \cdot G^{\prime}(Y)-G(Y) ; \\
& v=\sqrt{\frac{g l}{G^{\prime}(Y)}} .
\end{aligned}
$$

The reciprocal curve cusps correspond to the initial curve, $G(\bar{Y})$, inflection points.

It should be shown that in cases of general position a threefold stationary state with $\theta=0, v=v_{k p}$ corresponds to a

$G(\bar{Y})$ function inflection point at the origin (rectilinear motion mode stability loss occurs due to a pair of unstable stationary states emerging: with $\theta=0, v=v_{k p}=\left(\frac{\bar{k}_{1} \cdot \bar{k}_{2}}{\bar{k}_{1}-\bar{k}_{2}} g l\right)^{1 / 2}$ or a pair of stable states appearing).

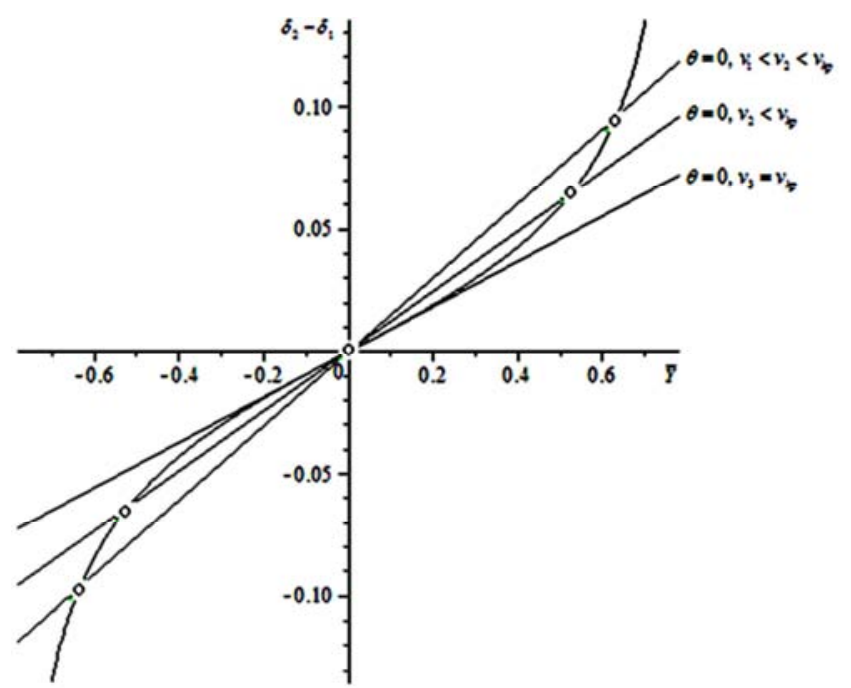

(a) illustration of stability loss mechanism in a crew model straight steady state of movement with oversteer

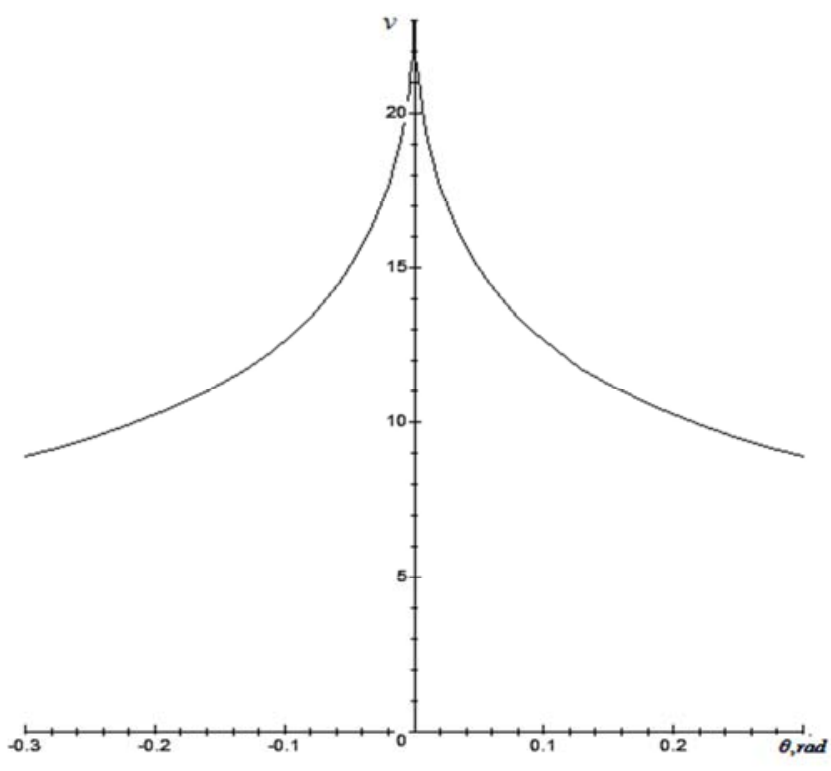

(b) the corresponding critical set of parameters (the bifurcation set) is a typical cross-section features "assembly"

Figure 6. Divergent stability loss in a straight mode of movement in case of one point curve inflection $G(\bar{Y})$

In case of slip forces approximation as a monotonic dependence (9):

$$
\bar{Y}_{i}\left(\delta_{i}\right)=\bar{k}_{i} \delta_{i}\left(1+\bar{k}_{i}^{2} \delta_{i}^{2} / \phi_{i}^{2}\right)^{-1 / 2},
$$

the first two function expansion terms, $\left(\delta_{2}-\delta_{1}\right)=G(\bar{Y})$, look as:

$$
G(\bar{Y})=\left(\bar{k}_{2}^{-1}-\bar{k}_{1}^{-1}\right) \bar{Y}+1 / 2\left(\bar{k}_{2}^{-1} \phi_{2}^{-2}-\bar{k}_{1}^{-1} \phi_{1}^{-2}\right) \bar{Y}^{3}+\ldots,
$$

and equation (7) is going to be represented as a third degree polynomial (11) (defines the stationary states manifold within rectilinear motion mode small neighbourhood):

$$
\left(\bar{k}_{2}^{-1}-\bar{k}_{1}^{-1}\right) \bar{Y}+1 / 2\left(\bar{k}_{2}^{-1} \phi_{2}^{-2}-\bar{k}_{1}^{-1} \phi_{1}^{-2}\right) \bar{Y}^{3}=g l / v^{2} \cdot \bar{Y}-\theta .
$$

Analyzing of equation (11) solutions number within the $v=v_{k p}$ critical velocity small neighbourhood should be done (it's suggested that $\bar{k}_{1}>\bar{k}_{2}, \theta=0$ ). Equation (11) can be viewed as follows with these suggestions:

$$
g l / v_{k p}^{2} \cdot\left(1-\frac{v_{k p}^{2}}{v^{2}}\right) \bar{Y}+1 / 2\left(\frac{\bar{k}_{1} \phi_{1}^{2}-\bar{k}_{2} \phi_{2}^{2}}{\bar{k}_{1} \phi_{1}^{2} \cdot \bar{k}_{2} \phi_{2}^{2}}\right) \bar{Y}^{3}=0,
$$

Then there are three stationary states (a stable one and two unstable ones) there with $v<v_{k p}$ and $\bar{k}_{1} \phi_{1}^{2}>\bar{k}_{2} \phi_{2}^{2}$, and there is one stationary state (unstable) there with $v>v_{k p}$ and $\bar{k}_{1} \phi_{1}^{2}>\bar{k}_{2} \phi_{2}^{2}$. If $\bar{k}_{1} \phi_{1}^{2}<\bar{k}_{2} \phi_{2}^{2}$, then there are three stationary states (an unstable one and two stable ones) with $v>v_{k p}$ - the case of a pair of stable stationary states emerging. This way, 
in general position cases $\left(\bar{k}_{1} \phi_{1}^{2} \neq \bar{k}_{2} \phi_{2}^{2}\right)$ with $\theta=0, v=v_{k p}$ a threefold stationary state is implemented at the origin; if the $\bar{k}_{1} \phi_{1}^{2}=\bar{k}_{2} \phi_{2}^{2}$ correlation is valid, it's necessary to involve $G(\bar{Y})$ function quintic expansion terms inclusively for further analysis. The bifurcation set (more precisely, its part within the small enough $\theta=0, v=v_{k p}$ point neighbourhood) is obtained as a semi-cubical parabola - a points locus consistent with theDiscrim3 $(\theta, v)=0$ equation where Discrim3 $(\theta, v)$ is equation (11) discriminant. Control parameters matching the rectilinear threefold stationary state $\left(\theta=0, v=v_{k p}\right)$ correspond to a semi-cubical parabola cusp, twofold circular stationary states with large enough radius of curvature value correspond to the rest of the discriminant manifold points. Three stationary states on an equilibrium surface correspond to the surface of the system (1) equilibrium states which in this case possesses and "assembly" feature -a wedge-shaped parameters plane part formed with the semi-cubical parabola, one stationary state corresponds to the rest of the parameters plane points. An expansion of parameter space where additional critical parameter sets are possible (matching the $\bar{k}_{1} \phi_{1}^{2}=\bar{k}_{2} \phi_{2}^{2}$ condition is also necessary along with $\theta=0, v=v_{k p}$ ) is required to implement a higher rank vehicle symmetrical design feature - "a butterfly" (corresponds to the fivefold stationary state).

Figure $7(b)$ depicts bifurcation set changes with varying $\phi_{1}$ "internal" parameter which is explained by additional inflection points appearing on the $G(\bar{Y})$ curve (figure7, $a$ )).

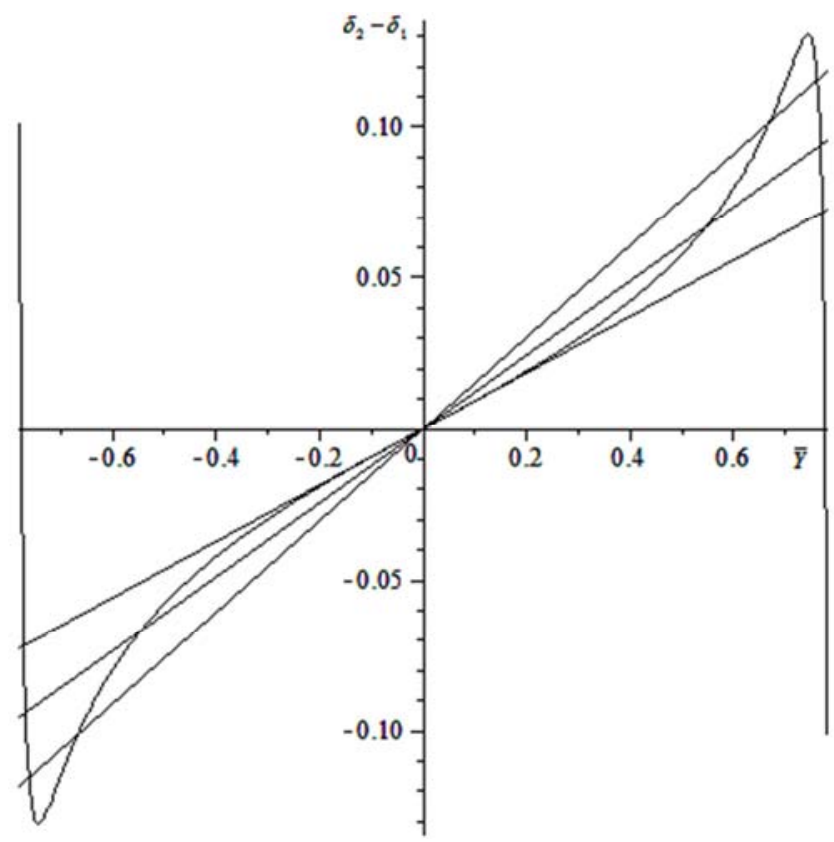

(a) $\mathrm{v}=18,20,23 ; \varphi_{1}=0,79$ an oversteering vehicle model straight stationary mode loss of stability mechanism illustration

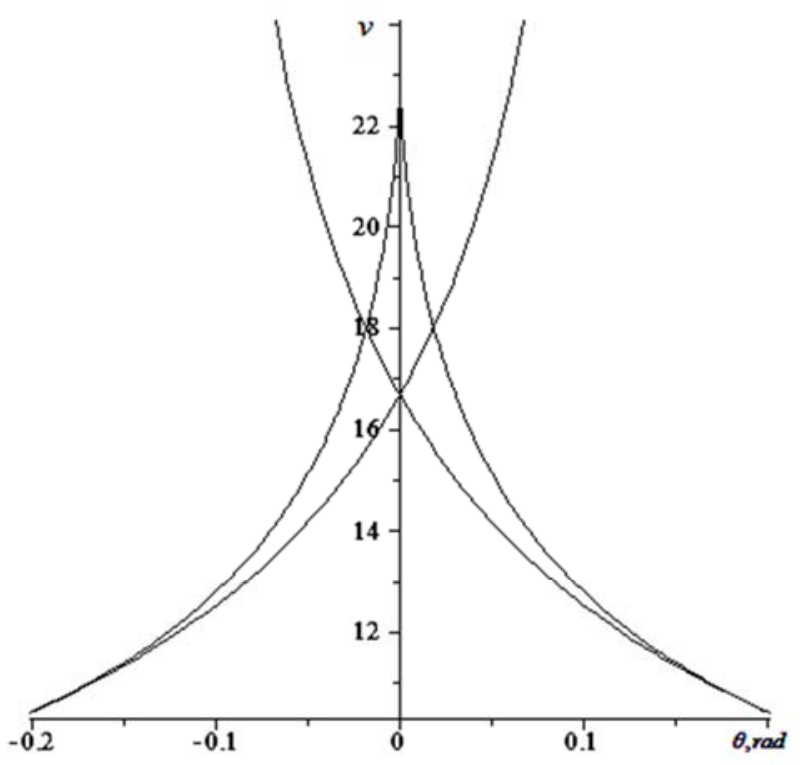

(b) $\varphi_{1}=0,79$ the corresponding critical set of parameters (the bifurcation set) represents a typical cross-section features "Butterfly"

Figure 7. Divergent stability loss in a straight mode of movement in case of three points curve inflection $G(\bar{Y})$

Moving to the analysis of circular stationary states consistent with handling curve divergent stability loss. As the case under studying is a special case of a circular stationary state stability loss this gives a possibility to define common point (if there are any there) when merging a stability (bifurcation set) diagram and a handling curve diagram on the same coordinate plane, in other case, divergent stability loss is uncharacteristic of stationary states corresponding to the handling curve.

A condition of common handling curve and bifurcation set points presence can be presented in an analytical form, this way, from the (8) and (6) correlation system (12) follows:

$$
\begin{gathered}
\theta=G^{\prime}(\bar{Y}) \cdot \bar{Y}-G(\bar{Y}), \\
\Rightarrow \quad G^{\prime}(\bar{Y}) \cdot \bar{Y}=l / R . \\
\theta=l / R-G(\bar{Y}) .
\end{gathered}
$$

It can be concluded from the "geometric" stability criterion that 1) circular stationary states corresponding to the handling curve stability loss relates to the $G^{\prime}(\bar{Y}) \cdot \bar{Y}<l / R$ inequation breaking.

Solving equation (12) for the $v$ parameter a critical velocity speed when moving along a circular path with the radius of curvature of $\mathrm{R}=30,5 \mathrm{~m}: v_{R=30,5}^{+}=13,17 \mathrm{~m} / \mathrm{s}$ (fi1:=0,8); $\quad v_{R=30,5}^{+}=13,86 \mathrm{~m} / \mathrm{s} ; \quad v_{R=30,5}^{+}=13,44 \quad \mathrm{~m} / \mathrm{s}$ (fi1: $=0,79$ ) is obtained.

With the front axle friction coefficient of fil: $=0,8$ an instability area corresponding to the handling curve (represented as a dashed curve at figure 8 ) is to the left and above its contact point $(\mathrm{v}=13,17 \mathrm{~m} / \mathrm{s})$ with the bifurcation set 
(continuous curve) in case of fi1: $=0,79$ an instability interval according to the velocity parameter is $(13,44 \mathrm{~m} / \mathrm{s}-14,86$ $\mathrm{m} / \mathrm{s}$ ), an area between two handling curve contact points with bifurcation curve (the dashed curve) corresponds to it (figure 9); a stable motion mode correspond to the rest of the handling curve points.

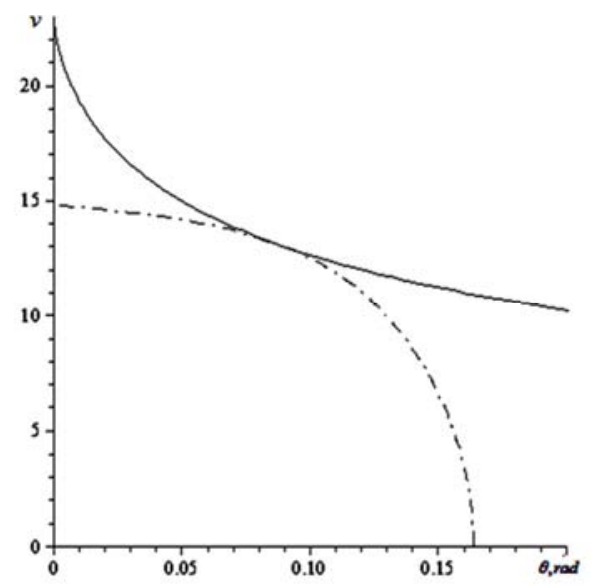

(a)

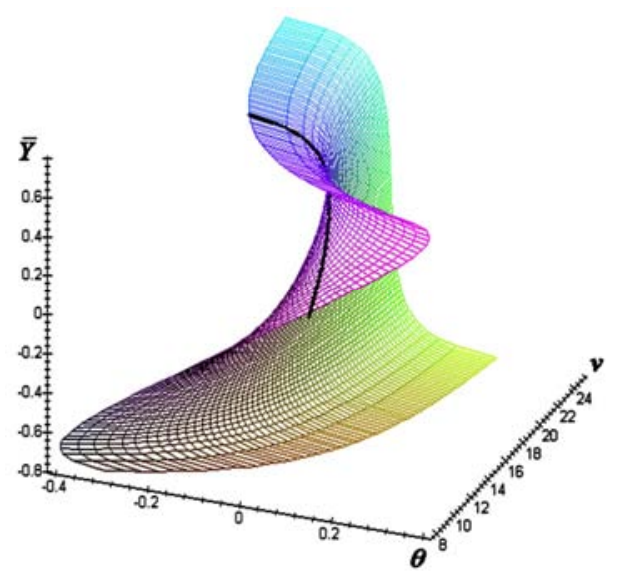

(b)

Figure 8. Definition of control parameters in divergent stability loss of stationary modes corresponding to the understeer curve using the bifurcation diagram.

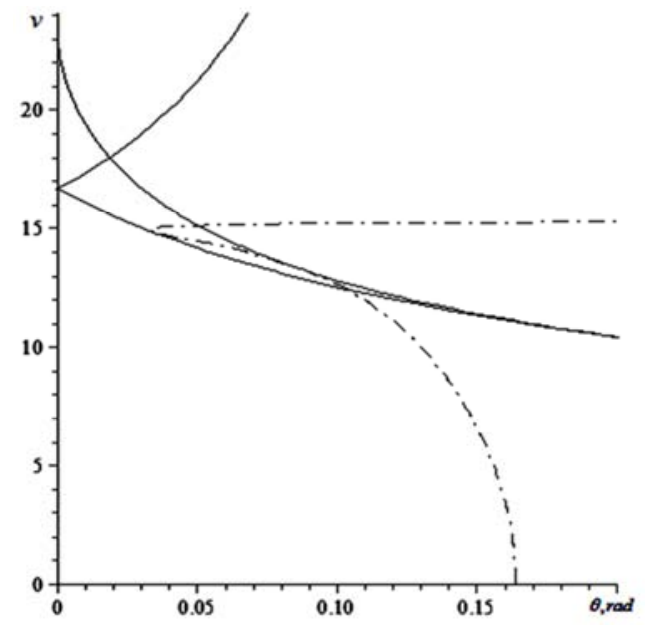

(a)

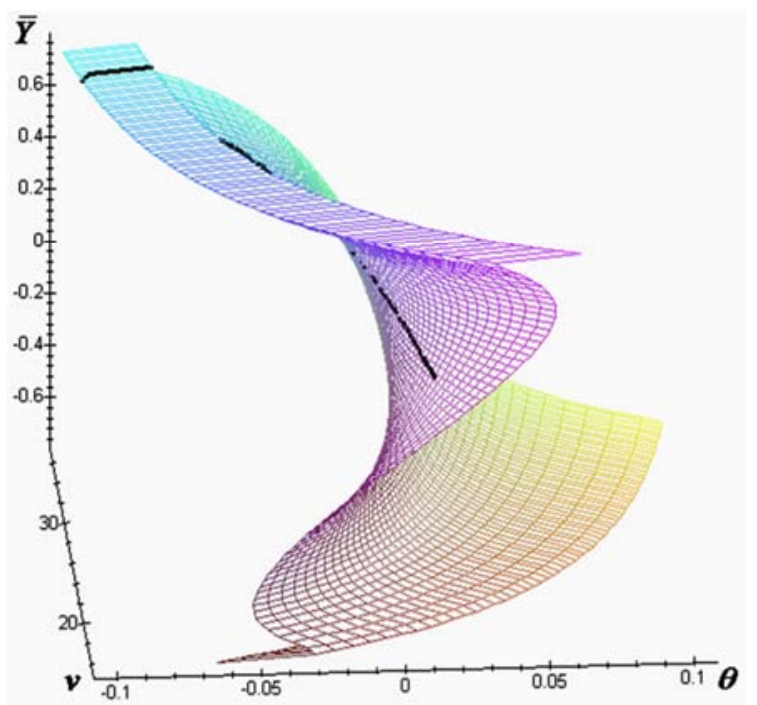

(b)

Figure 9. Definition of control parameters in divergent stability loss of stationary modes corresponding to the understeer curve, using the bifurcation diagram when $\varphi_{1}=0,79$

To conclude with, an additional stationary states pair appears (at the bottom contact point) in transverse handling curve with bifurcation set intersection points (figure 9) as well as it disappears (at the top point) which doesn't influence the main state stability with the fixed Ackermann angle, and the main state stability properties divergent change occurs at contact points (of non-transverse intersection): at the first contact point (the bottom one) - stability is lost; at the second (the top one) - stability properties are restored.

It's possible to define the corresponding path for which a divergent stability loss (or stability properties restoring) occurs radius of curvature from the velocity parameters values corresponding to divergent stability change set with equation (8). Indeed, the critical velocity value is defined as a specific side acceleration function, $v=v(\bar{Y})$, where $\bar{Y}=\frac{v^{2}}{g R}$, and from this the required dependency is found $R(\bar{Y})=\frac{v^{2}(\bar{Y})}{g \bar{Y}}$. It's natural to present the $R=R(v)$ dependency in a parametric form further on:

$$
\begin{aligned}
& v=\left(g l / G^{\prime}(\bar{Y})\right)^{1 / 2}, \\
& R=l / G^{\prime}(\bar{Y}) \cdot \bar{Y}
\end{aligned}
$$

A corresponding $R=R(v)$ dependency diagram (a continuous curve consistent with the fil: $=0,8$ value) a vertical asymptote at the $v=v_{k p}=\left(\frac{\bar{k}_{1} \cdot \bar{k}_{2}}{\bar{k}_{1}-\bar{k}_{2}} g l\right)^{1 / 2}$ point (figure $10)$, an $R=R(v)$ dependency diagram is represented as a dashed line (whenfil: $=0,79$ ), an instability interval for the velocity parameter $(13,44 \mathrm{~m} / \mathrm{s}-14,86 \mathrm{~m} / \mathrm{s})$ which co-insides with the instability interval received before (figure 9) corresponds to the $\mathrm{R}=30,5 \mathrm{~m}$ value; a border consisting of 
discrete points is defined with the maximum specific side acceleration value on the handling curve,

$$
\bar{Y}_{\max }=\frac{v^{2}}{g R} \leq \min \left\{\phi_{1}, \phi_{2}\right\} \text {, then } v_{R}^{\max } \leq \sqrt{\phi_{1} g R} .
$$

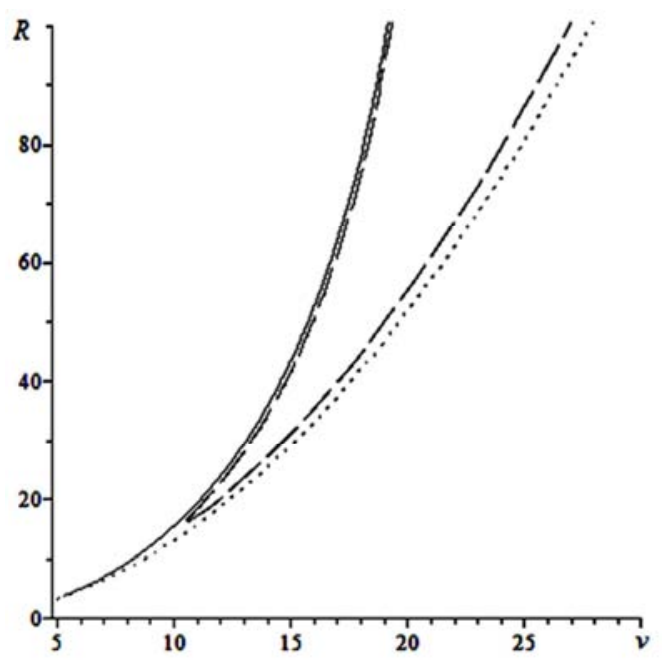

Figure 10. Dependence of vehicle longitudinal velocity center of mass trajectory curvature minimum radius when $\varphi 1=0,79$ - dashed line and when $\varphi 1=0,8$ solid line

As it could be expected, in practice, there is no considerable minimum radius of curvature changing because of insignificant $\varphi 1$ parameter varying there.

When the $\bar{k}_{1}>\bar{k}_{2}, \bar{k}_{1} \phi_{1}^{2}<\bar{k}_{2} \phi_{2}^{2}$ conditions are fulfilled (the case of fil $=0,7$ is reviewed) a divergent stability loss on the handling curve doesn't occur, if the path radius of curvature doesn't exceed the $183 \mathrm{~m}$ value.

Figure 11 depicts divergent stability loss absence on the handling curve with $R=100 \mathrm{~m}, R=160 \mathrm{~m}$.

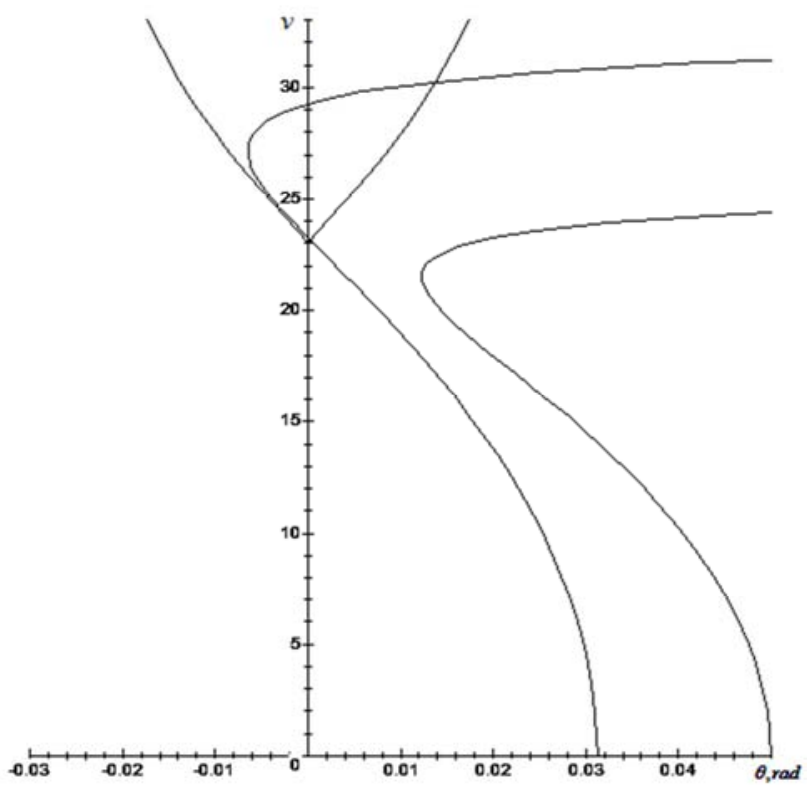

(a) $\mathrm{R}=100 \mathrm{~m}, 160 \mathrm{~m} ; \varphi_{1}=0,7, \varphi_{2}=0,8$

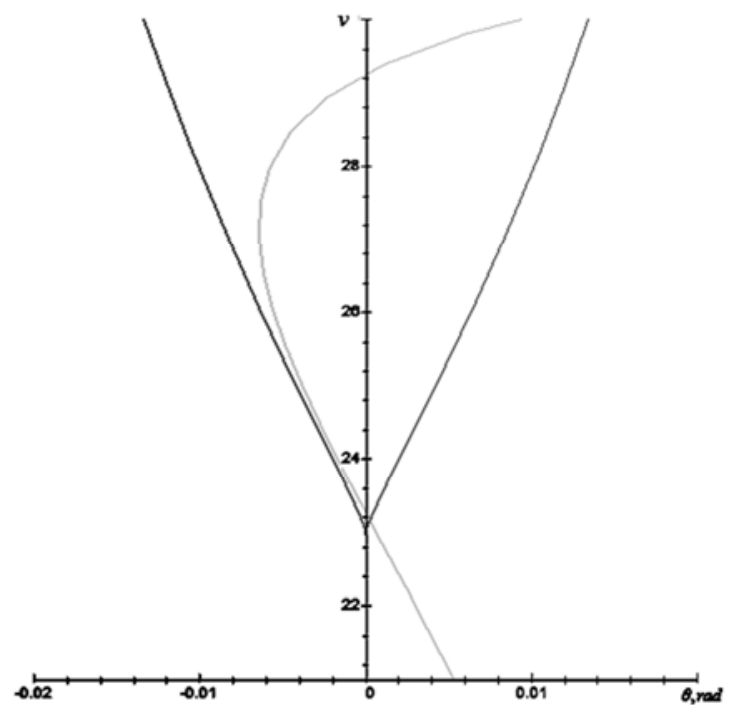

(b)

Figure 11. Analysis of the possible divergent stability loss of the stationary modes corresponding to the understeer curve based on the bifurcation diagram.

A critical radius of curvature value of $R=183 \mathrm{~m}$ is defined from figure 12: an $R=R(v)$ area of divergent stability loss (set with a continuous curve). The radius of curvature $R=183 m$ value corresponds to the divergent instability area top, the left instability area border has a vertical asymptote of:

$$
v=v_{k p}=\left(\frac{\overline{k_{1}} \cdot \bar{k}_{2}}{\bar{k}_{1}-\bar{k}_{2}} g l\right)^{1 / 2} .
$$

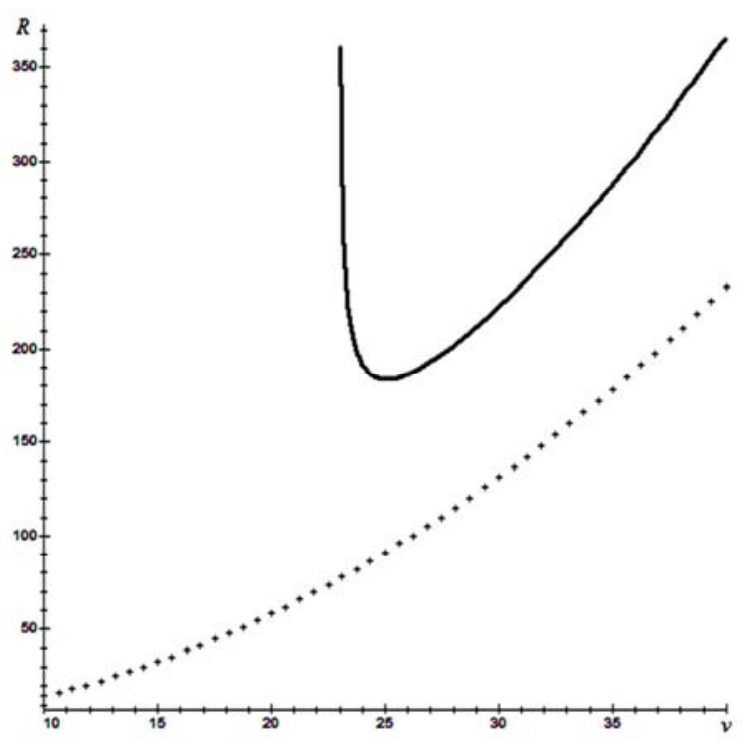

Figure 12. The smallest possible radii of curvature in case of $\varphi_{1}=0,7$ : inner region of a continuous curve corresponds to the area of divergence instability; the outer boundary (the discrete curve) is determined by the corresponding maximum value of the specific lateral acceleration $\bar{Y}$ in which the understeer curve is defined

Minimum possible radii of curvature (figure 12) for the case of fil $1:=0,7$ : a continuous curve internal area corresponds 
to the divergent instability area; the external border (a discrete response) is defined with the corresponding $\bar{Y}$ maximum specific side acceleration value(figure11) and handling curve defined along.

Thus, fil parameter reducing to 0,7 causes considerable minimum possible radii of curvature reducing and a new quality - a possibility of quite large radius of curvature circular stationary states implementation with velocity values matching the $v_{R}^{\max } \leq \sqrt{\phi_{1} g R}$ condition (the $R=R(v)$ border is represented a discrete response in this case).

\section{The Results of Analyzing}

The results of analyzing a vehicle with front steering axle model divergent stability loss are given below. A rear axle slip angle is adjusted increasingly at the $K_{\omega} \omega$ value:

$$
\delta_{2}=K_{\omega} \omega+\operatorname{arctg} \frac{-u+b \omega}{v} .
$$

Equations (13), (14) correspond to equations (6) and (7) accordingly:

$$
\begin{aligned}
& \theta=l / R+K_{\omega} \cdot(\bar{Y} \cdot g / R)^{1 / 2}-G(\bar{Y}) \\
& G(\bar{Y})=g l / v^{2} \cdot \bar{Y} \cdot\left(K_{\omega} \cdot v / l+1\right)-\theta .
\end{aligned}
$$

At figure 13, (a) stationary states manifold divergent stability loss diagrams with control ratio values are depicted. Handling curves $(R=30,5 \mathrm{~m})$ are also represented at figure 13 , (a) - critical velocity values correspond to the contact points $v_{R=30,5}^{+}=13,17 \mathrm{~m} / \mathrm{s} ; \quad v_{R=30,5}^{+}=13,64 \quad \mathrm{~m} / \mathrm{s}$; $v_{R=30,5}^{+}=13,85 \mathrm{~m} / \mathrm{s}$.

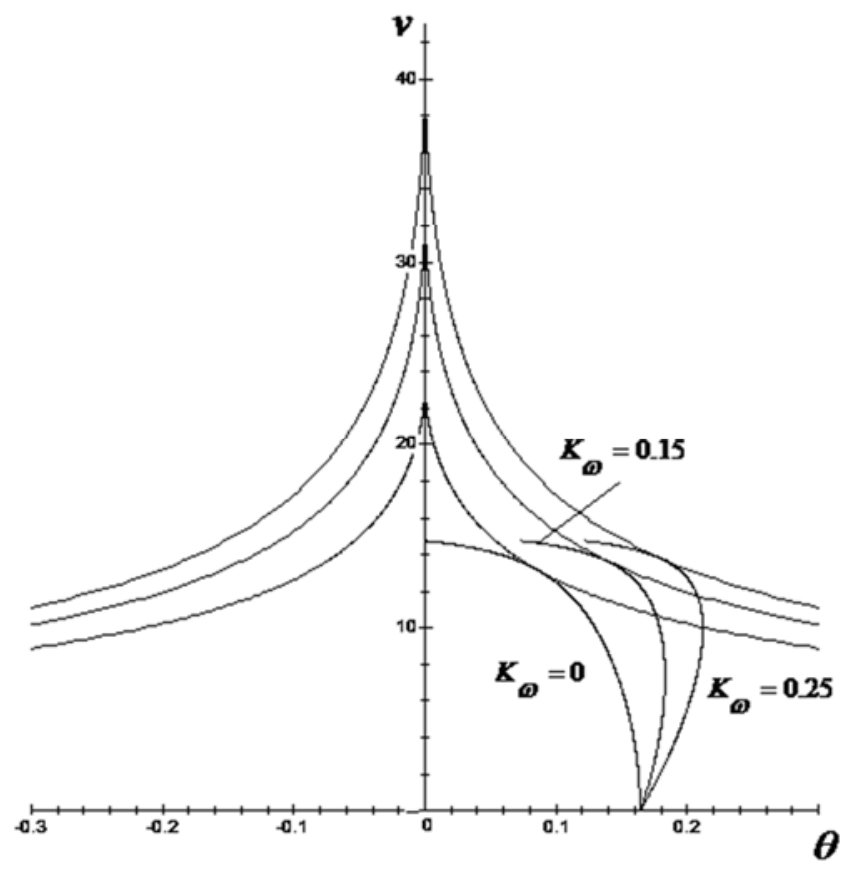

(a) diagrams of divergence buckling

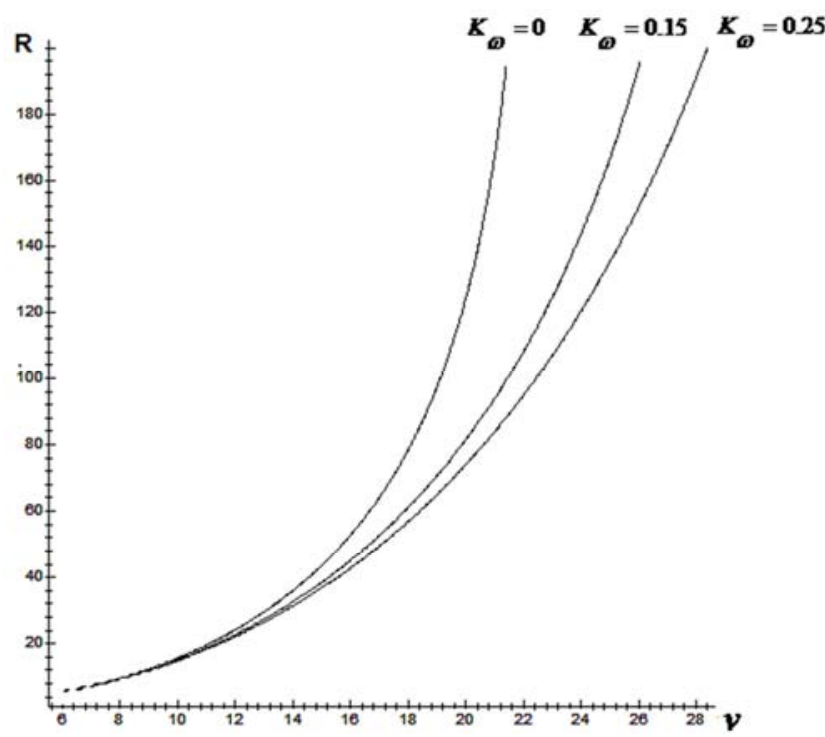

(b) the minimum radius of curvature of the trajectory for different values of the longitudinal velocity

Figure 13. Features of stability and understeer wheeled vehicle model with the additional control of the rear axle wheels $\left(K_{\omega}=0 ; 0,15 ; 0,25\right)$.

Analyzing results received based on the suggested twoaxle vehicle model stability and handling researchingapproach points at considerable effectiveness of introducing rear axle steering into design, in particular: a model with oversteering rectilinear motion critical velocity increases considerably; minimum possible motion circular stationary states radii of curvature are reduced; a maximum possible velocity in circular paths with quite large radii of curvature increases; a control ration effective value is within the $K_{\omega}=0,15-0,2$ range.

\section{Conclusions}

A grapho-analytical approach towards two-axle vehicle model entire stationary states manifold divergent stability loss analyzing based on the ideas of bifurcation analysis is revealed in the work.

The peculiarity of this approach is getting divergent stability loss conditions and minimum possible radii of curvature corresponding to circular stationary states without any previous defining of the stationary states manifold itself.

This approach is going to be convenient for evaluating the advisability of various design solutions aimed at dynamic qualities improvement.

\section{References}

[1] Gillespie, Thomas D. Fundamentals of Vehicle Dynamics. Society of Automotive Engineers, Inc., 1992, p. 470.

[2] Kravchenko, A. P., Verbitskii V. G., Zagorodnov M. I., Bannikov V. O., Sakno O. P., Efimenko A. N., TurchinaN. A. On the problem of the steerability analysis of the automobile non-linear model. NaukovivistiDalivskogouniversitetu. Electronenaukovefakhovevidanua, 2010, №1 (in Russian). 
[3] Yu, N., SaravananMuthiah S., Kulakowski, B. T. Analysis of steady-state handling behavior of a transit bus. 9th International Symposium on Heavy Vehicle Weights and Dimensions, June 18-22, 2006, Pennsylvania State University, State College, Pennsylvania, 01388838.

[4] Liu Lil, Shi Shuming, ShenShuiwen, Chu Jiangwei. Vehicle Planar Motion Stability Study for Tyres Working in Extremely Nonlinear Region. Chinese journal of mechanical engineering, Vol. 23, No. 2, 2010.

[5] Vladimir, Sakhno, Alexander, Kravchenko, Andrey, Kostenko, Vladimir, Verbitskii Influence of hauling force on firmnessof plural stationary motions of passenger car model TEKA Kom. Mot. iEnerg. Roln. OL PAN, 2011, 11B, pp. 147-155.

[6] Pevsner, J. M. Theory of stability of automobile. Moscow: Mashisdat, 1947, p. 156.

[7] Verbitskii, V., Danilenko, E., Nowak, A., Sitarz, M. Introduction in the Stability Theory of the Wheel Vehicles and Railway. Donetsk department: Veber, 2007, p. 255.

[8] Pacejka, H. B. Tyre factors and vehicle handling. Delf Univ. Technol, 1978,№ 108, p. 31.

[9] Fabio, Della Rossaa, GiampieroMastinub, Carlo Piccardia. Bifurcation analysis of an automobile model negotiating a curve. Vehicle System Dynamics, Vol. 50, No. 10, 2012, pp. $1539-1562$.

[10] Arnold, V. I. Catastrophe Theory. Moscow: Nauka, 1990, p. 128.

[11] Poston, T., Stewart, I. Catastrophe Theory AndIts Applications. Moscow: Mir, 1980, p. 607.
[12] Verbitskii, V. G. Bifurcation sets and catastrophes in manifold of the steady states of pneumowheel vehicles. Kiev: Pricl. Mech, Vol. 31, 13, 1995, pp. 89-95.

[13] Shinohara, Y. A geometric method for the numerical solution of non-linear equations and its application to non-linear oscillations. Publ. Res. Inst. Math. Sci., Kyoto Univ. 8, 1, 1972, pp. 13-42.

[14] Holodniok, M., Klic, A., Kubicek, M., Marek, M. Methods of Analysing Non-linear Dynamic Systems. Moscow: Mir, 1991, p. 368 .

[15] Rokar, I. Instability in Mechanics Automobiles. Airplanes. Suspension Bridges. Moscow: IL, 1959, p. 288 (Russian translation).

[16] [16]Ellis, J. R. Vehicle Dynamics. Moscow: Mashinostroenie, 1975, p. 216. (Russian translation).

[17] Lobas, L. G., Verbitskii, V. G. Qualitative and Analytical Methods in the Dynamics of Wheel Machines. Kiev: NaukovaDumka, 1990 (in Russian).

[18] Troger, H., Zeman, K. A nonlinear analysis of the generic types of loss of stability of the steady state motion of the tractor - semitrailer, Vehicle System Dynamics, Vol. 13, № 4, 1984, pp. 161-172.

[19] Verbitskii, V. G., Lobas, L. G. Method of determination of the special points and their character. Applied mathematics and mechanics, 1981, № 45 (5), pp. 944-948.

[20] Bruce, J., Giblin, P. Curves and Singularities. Moscow: Mir, 1988, p. 262. 